\title{
Difference and Difference Quotient. Part III
}

\author{
Xiquan Liang \\ Qingdao University of Science \\ and Technology \\ China
}

\author{
Ling Tang \\ Qingdao University of Science \\ and Technology \\ China
}

Summary. In this article, we give some important theorems of forward difference, backward difference, central difference and difference quotient and forward difference, backward difference, central difference and difference quotient formulas of some special functions.

MML identifier: $\underline{D_{F} F_{-} 3}$, version: $\underline{7.11 .04 \quad 4.130 .1076}$

The terminology and notation used in this paper have been introduced in the following papers: [6], [2], [1], [4], [11], [7], [5], [8], [12], [9], [10], and [3].

We follow the rules: $n, m$ are elements of $\mathbb{N}, h, k, r, r_{1}, r_{2}, x, x_{0}, x_{1}, x_{2}, x_{3}$ are real numbers, and $f, f_{1}, f_{2}$ are functions from $\mathbb{R}$ into $\mathbb{R}$.

Next we state a number of propositions:

(1) $\left(\delta_{h}[f]\right)(x)=\left(\Delta_{\frac{h}{2}}[f]\right)(x)-\left(\Delta_{-\frac{h}{2}}[f]\right)(x)$.

(2) $\quad\left(\Delta_{-\frac{h}{2}}[f]\right)(x)=-\left(\nabla_{\frac{h}{2}}[f]\right)(x)$.

(3) $\quad\left(\delta_{h}[f]\right)(x)=\left(\nabla_{\frac{h}{2}}[f]\right)(x)-\left(\nabla_{-\frac{h}{2}}[f]\right)(x)$.

(4) $\left(\vec{\Delta}_{h}\left[r f_{1}+f_{2}\right]\right)(n+1)(x)=r \cdot\left(\vec{\Delta}_{h}\left[f_{1}\right]\right)(n+1)(x)+\left(\vec{\Delta}_{h}\left[f_{2}\right]\right)(n+1)(x)$.

(5) $\left(\vec{\Delta}_{h}\left[f_{1}+r f_{2}\right]\right)(n+1)(x)=\left(\vec{\Delta}_{h}\left[f_{1}\right]\right)(n+1)(x)+r \cdot\left(\vec{\Delta}_{h}\left[f_{2}\right]\right)(n+1)(x)$.

(6) $\left(\vec{\Delta}_{h}\left[r_{1} f_{1}-r_{2} f_{2}\right]\right)(n+1)(x)=r_{1} \cdot\left(\vec{\Delta}_{h}\left[f_{1}\right]\right)(n+1)(x)-r_{2} \cdot\left(\vec{\Delta}_{h}\left[f_{2}\right]\right)(n+$ 1) $(x)$.

(7) $\left(\vec{\Delta}_{h}[f]\right)(1)=\Delta_{h}[f]$.

(8) $\left(\vec{\nabla}_{h}\left[r f_{1}+f_{2}\right]\right)(n+1)(x)=r \cdot\left(\vec{\nabla}_{h}\left[f_{1}\right]\right)(n+1)(x)+\left(\vec{\nabla}_{h}\left[f_{2}\right]\right)(n+1)(x)$.

(9) $\left(\vec{\nabla}_{h}\left[f_{1}+r f_{2}\right]\right)(n+1)(x)=\left(\vec{\nabla}_{h}\left[f_{1}\right]\right)(n+1)(x)+r \cdot\left(\vec{\nabla}_{h}\left[f_{2}\right]\right)(n+1)(x)$.

(10) $\left(\vec{\nabla}_{h}\left[r_{1} f_{1}-r_{2} f_{2}\right]\right)(n+1)(x)=r_{1} \cdot\left(\vec{\nabla}_{h}\left[f_{1}\right]\right)(n+1)(x)-r_{2} \cdot\left(\vec{\nabla}_{h}\left[f_{2}\right]\right)(n+$ 1) $(x)$. 
(11) $\quad\left(\vec{\nabla}_{h}[f]\right)(1)=\nabla_{h}[f]$

(12) $\left(\vec{\nabla}_{h}\left[\left(\vec{\nabla}_{h}[f]\right)(m)\right]\right)(n)(x)=\left(\vec{\nabla}_{h}[f]\right)(m+n)(x)$.

(13) $\left(\vec{\delta}_{h}\left[r f_{1}+f_{2}\right]\right)(n+1)(x)=r \cdot\left(\vec{\delta}_{h}\left[f_{1}\right]\right)(n+1)(x)+\left(\vec{\delta}_{h}\left[f_{2}\right]\right)(n+1)(x)$.

(14) $\left(\vec{\delta}_{h}\left[f_{1}+r f_{2}\right]\right)(n+1)(x)=\left(\vec{\delta}_{h}\left[f_{1}\right]\right)(n+1)(x)+r \cdot\left(\vec{\delta}_{h}\left[f_{2}\right]\right)(n+1)(x)$.

(15) $\quad\left(\vec{\delta}_{h}\left[r_{1} f_{1}-r_{2} f_{2}\right]\right)(n+1)(x)=r_{1} \cdot\left(\vec{\delta}_{h}\left[f_{1}\right]\right)(n+1)(x)-r_{2} \cdot\left(\vec{\delta}_{h}\left[f_{2}\right]\right)(n+1)(x)$.

(16) $\left(\vec{\delta}_{h}[f]\right)(1)=\delta_{h}[f]$.

(17) $\quad\left(\vec{\delta}_{h}\left[\left(\vec{\delta}_{h}[f]\right)(m)\right]\right)(n)(x)=\left(\vec{\delta}_{h}[f]\right)(m+n)(x)$.

(18) If $\left(\vec{\Delta}_{h}[f]\right)(n)(x)=\left(\vec{\delta}_{h}[f]\right)(n)\left(x+\frac{n}{2} \cdot h\right)$, then $\left(\vec{\nabla}_{h}[f]\right)(n)(x)=$ $\left(\vec{\delta}_{h}[f]\right)(n)\left(x-\frac{n}{2} \cdot h\right)$.

(19) If $\left(\vec{\Delta}_{h}[f]\right)(n)(x)=\left(\vec{\delta}_{h}[f]\right)(n)\left(x+\frac{n-1}{2} \cdot h+\frac{h}{2}\right)$, then $\left(\vec{\nabla}_{h}[f]\right)(n)(x)=$ $\left(\vec{\delta}_{h}[f]\right)(n)\left(x-\frac{n-1}{2} \cdot h-\frac{h}{2}\right)$.

(20) $\Delta[f](x, x+h)=\frac{\left(\Delta_{h}[f]\right)(x)}{h}$.

(21) $\Delta[f](x-h, x)=\frac{\left(\nabla_{h}[f]\right)(x)}{h}$.

(22) $\Delta[f]\left(x-\frac{h}{2}, x+\frac{h}{2}\right)=\frac{\left(\delta_{h}[f]\right)(x)}{h}$.

(23) $\Delta[f]\left(x-\frac{h}{2}, x+\frac{h}{2}\right)=\frac{\left(\vec{\delta}_{h}[f]\right)(1)(x)}{h}$.

(24) If $h \neq 0$, then $\Delta[f](x-h, x, x+h)=\frac{\left(\vec{\delta}_{h}[f]\right)(2)(x)}{2 \cdot h \cdot h}$.

(25) $\Delta\left[f_{1}-f_{2}\right]\left(x_{0}, x_{1}\right)=\Delta\left[f_{1}\right]\left(x_{0}, x_{1}\right)-\Delta\left[f_{2}\right]\left(x_{0}, x_{1}\right)$.

(26) $\Delta\left[r f_{1}+f_{2}\right]\left(x_{0}, x_{1}\right)=r \cdot \Delta\left[f_{1}\right]\left(x_{0}, x_{1}\right)+\Delta\left[f_{2}\right]\left(x_{0}, x_{1}\right)$.

(27) $\Delta\left[r f_{1}-f_{2}\right]\left(x_{0}, x_{1}\right)=r \cdot \Delta\left[f_{1}\right]\left(x_{0}, x_{1}\right)-\Delta\left[f_{2}\right]\left(x_{0}, x_{1}\right)$.

(28) $\Delta\left[f_{1}+r f_{2}\right]\left(x_{0}, x_{1}\right)=\Delta\left[f_{1}\right]\left(x_{0}, x_{1}\right)+r \cdot \Delta\left[f_{2}\right]\left(x_{0}, x_{1}\right)$.

(29) $\Delta\left[f_{1}-r f_{2}\right]\left(x_{0}, x_{1}\right)=\Delta\left[f_{1}\right]\left(x_{0}, x_{1}\right)-r \cdot \Delta\left[f_{2}\right]\left(x_{0}, x_{1}\right)$.

(30) $\Delta\left[r_{1} f_{1}-r_{2} f_{2}\right]\left(x_{0}, x_{1}\right)=r_{1} \cdot \Delta\left[f_{1}\right]\left(x_{0}, x_{1}\right)-r_{2} \cdot \Delta\left[f_{2}\right]\left(x_{0}, x_{1}\right)$.

(31) $\left(\vec{\nabla}_{h}\left[f_{1} f_{2}\right]\right)(1)(x)=f_{1}(x) \cdot\left(\vec{\nabla}_{h}\left[f_{2}\right]\right)(1)(x)+f_{2}(x-h) \cdot\left(\vec{\nabla}_{h}\left[f_{1}\right]\right)(1)(x)$.

(32) If $x_{0}, x_{1}, x_{2}$ are mutually different, then $\Delta[f]\left(x_{0}, x_{1}, x_{2}\right)=$ $\Delta[f]\left(x_{0}, x_{2}, x_{1}\right)$.

In the sequel $S$ is a sequence of real sequences.

We now state a number of propositions:

(33) Suppose that for all natural numbers $n, i$ such that $i \leq n$ holds $S(n)(i)=$ $\left(\begin{array}{c}n \\ i\end{array}\right) \cdot\left(\vec{\nabla}_{h}\left[f_{1}\right]\right)(i)(x) \cdot\left(\vec{\nabla}_{h}\left[f_{2}\right]\right)\left(n-{ }^{\prime} i\right)(x-i \cdot h)$. Then $\left(\vec{\nabla}_{h}\left[f_{1} f_{2}\right]\right)(1)(x)=$ $\sum_{\kappa=0}^{1} S(1)(\kappa)$ and $\left(\vec{\nabla}_{h}\left[f_{1} f_{2}\right]\right)(2)(x)=\sum_{\kappa=0}^{2} S(2)(\kappa)$.

(34) $\left(\vec{\delta}_{h}\left[f_{1} f_{2}\right]\right)(1)(x)=f_{1}\left(x+\frac{h}{2}\right) \cdot\left(\vec{\delta}_{h}\left[f_{2}\right]\right)(1)(x)+f_{2}\left(x-\frac{h}{2}\right) \cdot\left(\vec{\delta}_{h}\left[f_{1}\right]\right)(1)(x)$.

(35) Suppose that for all natural numbers $n$, $i$ such that $i \leq n$ holds $S(n)(i)=\left(\begin{array}{l}n \\ i\end{array}\right) \cdot\left(\vec{\delta}_{h}\left[f_{1}\right]\right)(i)\left(x+\left(n-^{\prime} i\right) \cdot \frac{h}{2}\right) \cdot\left(\vec{\delta}_{h}\left[f_{2}\right]\right)\left(n-{ }^{\prime} i\right)\left(x-i \cdot \frac{h}{2}\right)$. Then $\left(\vec{\delta}_{h}\left[f_{1} f_{2}\right]\right)(1)(x)=\sum_{\kappa=0}^{1} S(1)(\kappa)$ and $\left(\vec{\delta}_{h}\left[f_{1} f_{2}\right]\right)(2)(x)=\sum_{\kappa=0}^{2} S(2)(\kappa)$.

(36) If for every $x$ holds $f(x)=\sqrt{x}$ and $x_{0} \neq x_{1}$ and $x_{0}>0$ and $x_{1}>0$, then $\Delta[f]\left(x_{0}, x_{1}\right)=\frac{1}{\sqrt{x_{0}}+\sqrt{x_{1}}}$. 
(37) Suppose for every $x$ holds $f(x)=\sqrt{x}$ and $x_{0}, x_{1}, x_{2}$ are mutually different and $x_{0}>0$ and $x_{1}>0$ and $x_{2}>0$. Then $\Delta[f]\left(x_{0}, x_{1}, x_{2}\right)=$ $-\frac{1}{\left(\sqrt{x_{0}}+\sqrt{x_{1}}\right) \cdot\left(\sqrt{x_{0}}+\sqrt{x_{2}}\right) \cdot\left(\sqrt{x_{1}}+\sqrt{x_{2}}\right)}$.

(38) Suppose for every $x$ holds $f(x)=\sqrt{x}$ and $x_{0}, x_{1}, x_{2}, x_{3}$ are mutually different and $x_{0}>0$ and $x_{1}>0$ and $x_{2}>0$ and $x_{3}>0$.

Then $\Delta[f]\left(x_{0}, x_{1}, x_{2}, x_{3}\right)=$ $\frac{\sqrt{x_{0}}+\sqrt{x_{1}}+\sqrt{x_{2}}+\sqrt{x_{3}}}{\left(\sqrt{x_{0}}+\sqrt{x_{1}}\right) \cdot\left(\sqrt{x_{0}}+\sqrt{x_{2}}\right) \cdot\left(\sqrt{x_{0}}+\sqrt{x_{3}}\right) \cdot\left(\sqrt{x_{1}}+\sqrt{x_{2}}\right) \cdot\left(\sqrt{x_{1}}+\sqrt{x_{3}}\right) \cdot\left(\sqrt{x_{2}}+\sqrt{x_{3}}\right)}$.

(39) If for every $x$ holds $f(x)=\sqrt{x}$ and $x>0$ and $x+h>0$, then $\left(\Delta_{h}[f]\right)(x)=\sqrt{x+h}-\sqrt{x}$.

(40) If for every $x$ holds $f(x)=\sqrt{x}$ and $x>0$ and $x-h>0$, then $\left(\nabla_{h}[f]\right)(x)=\sqrt{x}-\sqrt{x-h}$.

(41) If for every $x$ holds $f(x)=\sqrt{x}$ and $x+\frac{h}{2}>0$ and $x-\frac{h}{2}>0$, then $\left(\delta_{h}[f]\right)(x)=\sqrt{x+\frac{h}{2}}-\sqrt{x-\frac{h}{2}}$.

(42) If for every $x$ holds $f(x)=x^{2}$ and $x_{0} \neq x_{1}$, then $\Delta[f]\left(x_{0}, x_{1}\right)=x_{0}+x_{1}$.

(43) If for every $x$ holds $f(x)=x^{2}$ and $x_{0}, x_{1}, x_{2}$ are mutually different, then $\Delta[f]\left(x_{0}, x_{1}, x_{2}\right)=1$.

(44) If for every $x$ holds $f(x)=x^{2}$ and $x_{0}, x_{1}, x_{2}, x_{3}$ are mutually different, then $\Delta[f]\left(x_{0}, x_{1}, x_{2}, x_{3}\right)=0$.

(45) If for every $x$ holds $f(x)=x^{2}$, then $\left(\Delta_{h}[f]\right)(x)=2 \cdot x \cdot h+h^{2}$.

(46) If for every $x$ holds $f(x)=x^{2}$, then $\left(\nabla_{h}[f]\right)(x)=h \cdot(2 \cdot x-h)$.

(47) If for every $x$ holds $f(x)=x^{2}$, then $\left(\delta_{h}[f]\right)(x)=2 \cdot h \cdot x$.

(48) If for every $x$ holds $f(x)=\frac{k}{x^{2}}$ and $x_{0} \neq x_{1}$ and $x_{0} \neq 0$ and $x_{1} \neq 0$, then $\Delta[f]\left(x_{0}, x_{1}\right)=-\frac{k}{x_{0} \cdot x_{1}} \cdot\left(\frac{1}{x_{0}}+\frac{1}{x_{1}}\right)$.

(49) Suppose for every $x$ holds $f(x)=\frac{k}{x^{2}}$ and $x_{0} \neq 0$ and $x_{1} \neq 0$ and $x_{2} \neq 0$ and $x_{0}, x_{1}, x_{2}$ are mutually different. Then $\Delta[f]\left(x_{0}, x_{1}, x_{2}\right)=$ $\frac{k}{x_{0} \cdot x_{1} \cdot x_{2}} \cdot\left(\frac{1}{x_{0}}+\frac{1}{x_{1}}+\frac{1}{x_{2}}\right)$.

(50) If for every $x$ holds $f(x)=\frac{k}{x^{2}}$ and $x \neq 0$ and $x+h \neq 0$, then $\left(\Delta_{h}[f]\right)(x)=$ $\frac{(-k) \cdot h \cdot(2 \cdot x+h)}{\left(x^{2}+h \cdot x\right)^{2}}$.

(51) If for every $x$ holds $f(x)=\frac{k}{x^{2}}$ and $x \neq 0$ and $x-h \neq 0$, then $\left(\nabla_{h}[f]\right)(x)=$ $\frac{(-k) \cdot h \cdot(2 \cdot x-h)}{\left(x^{2}-x \cdot h\right)^{2}}$.

(52) If for every $x$ holds $f(x)=\frac{k}{x^{2}}$ and $x+\frac{h}{2} \neq 0$ and $x-\frac{h}{2} \neq 0$, then $\left(\delta_{h}[f]\right)(x)=\frac{-2 \cdot h \cdot k \cdot x}{\left(x^{2}-\left(\frac{h}{2}\right)^{2}\right)^{2}}$.

(53) $\Delta[$ (the function $\sin )$ (the function $\sin$ ) (the function $\sin )]\left(x_{0}, x_{1}\right)=$ $\frac{\frac{1}{2} \cdot\left(3 \cdot \cos \left(\frac{x_{0}+x_{1}}{2}\right) \cdot \sin \left(\frac{x_{0}-x_{1}}{2}\right)-\cos \left(\frac{3 \cdot\left(x_{0}+x_{1}\right)}{2}\right) \cdot \sin \left(\frac{3 \cdot\left(x_{0}-x_{1}\right)}{2}\right)\right)}{x_{0}-x_{1}}$.

(54) $\left(\Delta_{h}[\right.$ (the function $\sin )$ (the function $\left.\sin \right)$ (the function $\left.\left.\left.\sin \right)\right]\right)(x)=\frac{1}{2}$. $\left(3 \cdot \cos \left(\frac{2 \cdot x+h}{2}\right) \cdot \sin \left(\frac{h}{2}\right)-\cos \left(\frac{3 \cdot(2 \cdot x+h)}{2}\right) \cdot \sin \left(\frac{3 \cdot h}{2}\right)\right)$. 
(55) $\left(\nabla_{h}[\right.$ (the function $\sin )$ (the function $\left.\sin \right)$ (the function $\left.\left.\left.\sin \right)\right]\right)(x)=\frac{1}{2} \cdot$ $\left(3 \cdot \cos \left(\frac{2 \cdot x-h}{2}\right) \cdot \sin \left(\frac{h}{2}\right)-\cos \left(\frac{3 \cdot(2 \cdot x-h)}{2}\right) \cdot \sin \left(\frac{3 \cdot h}{2}\right)\right)$.

(56) $\left(\delta_{h}[(\right.$ the function $\sin )$ (the function $\sin )$ (the function $\left.\left.\left.\sin \right)\right]\right)(x)=\frac{1}{2} \cdot(3$. $\left.\cos x \cdot \sin \left(\frac{h}{2}\right)-\cos (3 \cdot x) \cdot \sin \left(\frac{3 \cdot h}{2}\right)\right)$.

(57) $\Delta[$ (the function $\cos )$ (the function $\cos$ ) (the function $\cos )]\left(x_{0}, x_{1}\right)=$ $-\frac{\frac{1}{2} \cdot\left(3 \cdot \sin \left(\frac{x_{0}+x_{1}}{2}\right) \cdot \sin \left(\frac{x_{0}-x_{1}}{2}\right)+\sin \left(\frac{3 \cdot x_{0}+3 \cdot x_{1}}{2}\right) \cdot \sin \left(\frac{3 \cdot x_{0}-3 \cdot x_{1}}{2}\right)\right)}{x_{0}-x_{1}}$.

(58) $\left(\Delta_{h}[\right.$ (the function cos) (the function $\cos ) \quad($ the function $\left.\left.\cos )\right]\right)(x)=$ $-\frac{1}{2} \cdot\left(3 \cdot \sin \left(\frac{2 \cdot x+h}{2}\right) \cdot \sin \left(\frac{h}{2}\right)+\sin \left(\frac{3 \cdot(2 \cdot x+h)}{2}\right) \cdot \sin \left(\frac{3 \cdot h}{2}\right)\right)$.

(59) $\left(\nabla_{h}[\right.$ (the function $\cos ) \quad($ the function $\cos ) \quad($ the function $\left.\left.\cos )\right]\right)(x)=$ $-\frac{1}{2} \cdot\left(3 \cdot \sin \left(\frac{2 \cdot x-h}{2}\right) \cdot \sin \left(\frac{h}{2}\right)+\sin \left(\frac{3 \cdot(2 \cdot x-h)}{2}\right) \cdot \sin \left(\frac{3 \cdot h}{2}\right)\right)$.

(60) $\left(\delta_{h}[\right.$ (the function cos) (the function $\cos )$ (the function $\left.\left.\left.\cos \right)\right]\right)(x)=$ $-\frac{1}{2} \cdot\left(3 \cdot \sin x \cdot \sin \left(\frac{h}{2}\right)+\sin (3 \cdot x) \cdot \sin \left(\frac{3 \cdot h}{2}\right)\right)$.

(61) If for every $x$ holds $f(x)=\frac{1}{\sin x}$ and $\sin x_{0} \neq 0$ and $\sin x_{1} \neq 0$, then $\Delta[f]\left(x_{0}, x_{1}\right)=-\frac{\frac{2 \cdot\left(\sin x_{1}-\sin x_{0}\right)}{\cos \left(x_{0}+x_{1}\right)-\cos \left(x_{0}-x_{1}\right)}}{x_{0}-x_{1}}$.

(62) If for every $x$ holds $f(x)=\frac{1}{\sin x}$ and $\sin x \neq 0$ and $\sin (x+h) \neq 0$, then $\left(\Delta_{h}[f]\right)(x)=-\frac{2 \cdot(\sin x-\sin (x+h))}{\cos (2 \cdot x+h)-\cos h}$.

(63) If for every $x$ holds $f(x)=\frac{1}{\sin x}$ and $\sin x \neq 0$ and $\sin (x-h) \neq 0$, then $\left(\nabla_{h}[f]\right)(x)=\frac{(-2) \cdot(\sin (x-h)-\sin x)}{\cos (2 \cdot x-h)-\cos h}$.

(64) If for every $x$ holds $f(x)=\frac{1}{\sin x}$ and $\sin \left(x+\frac{h}{2}\right) \neq 0$ and $\sin \left(x-\frac{h}{2}\right) \neq 0$, then $\left(\delta_{h}[f]\right)(x)=-\frac{2 \cdot\left(\sin \left(x-\frac{h}{2}\right)-\sin \left(x+\frac{h}{2}\right)\right)}{\cos (2 \cdot x)-\cos h}$.

(65) If for every $x$ holds $f(x)=\frac{1}{\frac{\cos x}{2 \cdot\left(\cos x_{1}-\cos x_{0}\right)}}$ and $x_{0} \neq x_{1}$ and $\cos x_{0} \neq 0$ and $\cos x_{1} \neq 0$, then $\Delta[f]\left(x_{0}, x_{1}\right)=\frac{\frac{2 \cdot\left(\cos x_{1}-\cos x_{0}\right)}{\cos \left(x_{0}+x_{1}\right)+\cos \left(x_{0}-x_{1}\right)}}{x_{0}-x_{1}}$.

(66) If for every $x$ holds $f(x)=\frac{1}{\cos x}$ and $\cos x \neq 0$ and $\cos (x+h) \neq 0$, then $\left(\Delta_{h}[f]\right)(x)=\frac{2 \cdot(\cos x-\cos (x+h))}{\cos (2 \cdot x+h)+\cos h}$.

(67) If for every $x$ holds $f(x)=\frac{1}{\cos x}$ and $\cos x \neq 0$ and $\cos (x-h) \neq 0$, then $\left(\nabla_{h}[f]\right)(x)=\frac{2 \cdot(\cos (x-h)-\cos x)}{\cos (2 \cdot x-h)+\cos h}$.

(68) If for every $x$ holds $f(x)=\frac{1}{\cos x}$ and $\cos \left(x+\frac{h}{2}\right) \neq 0$ and $\cos \left(x-\frac{h}{2}\right) \neq 0$, then $\left(\delta_{h}[f]\right)(x)=\frac{2 \cdot\left(\cos \left(x-\frac{h}{2}\right)-\cos \left(x+\frac{h}{2}\right)\right)}{\cos (2 \cdot x)+\cos h}$.

(69) Suppose for every $x$ holds $f(x)=\frac{1}{(\sin x)^{2}}$ and $x_{0} \neq x_{1}$ and $\sin x_{0} \neq 0$ and $\sin x_{1} \neq 0$. Then $\Delta[f]\left(x_{0}, x_{1}\right)=\frac{16 \cdot \cos \left(\frac{x_{1}+x_{0}}{2}\right) \cdot \sin \left(\frac{x_{1}-x_{0}}{2}\right) \cdot \cos \left(\frac{x_{1}-x_{0}}{2}\right) \cdot \sin \left(\frac{x_{1}+x_{0}}{2}\right)}{\left(\cos \left(x_{0}+x_{1}\right)-\cos \left(x_{0}-x_{1}\right)\right)^{2} \cdot\left(x_{0}-x_{1}\right)}$.

(70) If for every $x$ holds $f(x)=\frac{1}{(\sin x)^{2}}$ and $\sin x \neq 0$ and $\sin (x+h) \neq 0$, then $\left(\Delta_{h}[f]\right)(x)=\frac{16 \cdot \cos \left(\frac{2 \cdot x+h}{2}\right) \cdot \sin \left(\frac{-h}{2}\right) \cdot \cos \left(\frac{-h}{2}\right) \cdot \sin \left(\frac{2 \cdot x+h}{2}\right)}{(\cos (2 \cdot x+h)-\cos h)^{2}}$.

(71) If for every $x$ holds $f(x)=\frac{1}{(\sin x)^{2}}$ and $\sin x \neq 0$ and $\sin (x-h) \neq 0$, then 
$\left(\nabla_{h}[f]\right)(x)=\frac{16 \cdot \cos \left(\frac{2 \cdot x-h}{2}\right) \cdot \sin \left(\frac{-h}{2}\right) \cdot \cos \left(\frac{-h}{2}\right) \cdot \sin \left(\frac{2 \cdot x-h}{2}\right)}{(\cos (2 \cdot x-h)-\cos h)^{2}}$.

(72) If for every $x$ holds $f(x)=\frac{1}{(\sin x)^{2}}$ and $\sin \left(x+\frac{h}{2}\right) \neq 0$ and $\sin \left(x-\frac{h}{2}\right) \neq 0$, then $\left(\delta_{h}[f]\right)(x)=\frac{16 \cdot \cos x \cdot \sin \left(\frac{-h}{2}\right) \cdot \cos \left(\frac{-h}{2}\right) \cdot \sin x}{(\cos (2 \cdot x)-\cos h)^{2}}$.

(73) Suppose for every $x$ holds $f(x)=\frac{1}{(\cos x)^{2}}$ and $x_{0} \neq x_{1}$ and $\cos x_{0} \neq 0$ and $\cos x_{1} \neq 0$. Then $\Delta[f]\left(x_{0}, x_{1}\right)=\frac{\frac{(-16) \cdot \sin \left(\frac{x_{1}+x_{0}}{2}\right) \cdot \sin \left(\frac{x_{1}-x_{0}}{2}\right) \cdot \cos \left(\frac{x_{1}+x_{0}}{2}\right) \cdot \cos \left(\frac{x_{1}-x_{0}}{2}\right)}{\left(\cos \left(x_{0}+x_{1}\right)+\cos \left(x_{0}-x_{1}\right)\right)^{2}}}{x_{0}-x_{1}}$.

(74) If for every $x$ holds $f(x)=\frac{1}{(\cos x)^{2}}$ and $\cos x \neq 0$ and $\cos (x+h) \neq 0$, then $\left(\Delta_{h}[f]\right)(x)=\frac{(-16) \cdot \sin \left(\frac{2 \cdot x+h}{2}\right) \cdot \sin \left(\frac{-h}{2}\right) \cdot \cos \left(\frac{2 \cdot x+h}{2}\right) \cdot \cos \left(\frac{-h}{2}\right)}{(\cos (2 \cdot x+h)+\cos h)^{2}}$.

(75) If for every $x$ holds $f(x)=\frac{1}{(\cos x)^{2}}$ and $\cos x \neq 0$ and $\cos (x-h) \neq 0$, then $\left(\nabla_{h}[f]\right)(x)=\frac{(-16) \cdot \sin \left(\frac{2 \cdot x-h}{2}\right) \cdot \sin \left(\frac{-h}{2}\right) \cdot \cos \left(\frac{2 \cdot x-h}{2}\right) \cdot \cos \left(\frac{-h}{2}\right)}{(\cos (2 \cdot x-h)+\cos h)^{2}}$.

(76) If for every $x$ holds $f(x)=\frac{1}{(\cos x)^{2}}$ and $\cos \left(x+\frac{h}{2}\right) \neq 0$ and $\cos \left(x-\frac{h}{2}\right) \neq 0$, then $\left(\delta_{h}[f]\right)(x)=\frac{(-16) \cdot \sin x \cdot \sin \left(\frac{-h}{2}\right) \cdot \cos x \cdot \cos \left(\frac{-h}{2}\right)}{(\cos (2 \cdot x)+\cos h)^{2}}$.

(77) Suppose $x_{0} \in \operatorname{dom}\left(\right.$ the function tan) and $x_{1} \in$ dom(the function $\tan )$. Then $\Delta[$ (the function $\tan$ ) (the function $\sin )]\left(x_{0}, x_{1}\right)=$ $\frac{\left(\frac{1}{\cos x_{0}}-\cos x_{0}-\frac{1}{\cos x_{1}}\right)+\cos x_{1}}{x_{0}-x_{1}}$.

(78) Suppose that

(i) for every $x$ holds $f(x)=(($ the function tan) (the function $\sin ))(x)$,

(ii) $\quad x \in \operatorname{dom}$ (the function tan), and

(iii) $x+h \in \operatorname{dom}$ (the function tan).

Then $\left(\Delta_{h}[f]\right)(x)=\left(\frac{1}{\cos (x+h)}-\cos (x+h)-\frac{1}{\cos x}\right)+\cos x$.

(79) Suppose that

(i) for every $x$ holds $f(x)=(($ the function tan) (the function sin) $)(x)$,

(ii) $x \in \operatorname{dom}$ (the function tan), and

(iii) $\quad x-h \in \operatorname{dom}$ (the function $\tan$ ).

Then $\left(\nabla_{h}[f]\right)(x)=\left(\frac{1}{\cos x}-\cos x-\frac{1}{\cos (x-h)}\right)+\cos (x-h)$.

(80) Suppose that

(i) for every $x$ holds $f(x)=(($ the function tan) (the function sin) $)(x)$,

(ii) $\quad x+\frac{h}{2} \in \operatorname{dom}$ (the function tan), and

(iii) $\quad x-\frac{h}{2} \in \operatorname{dom}$ (the function $\tan$ ).

Then $\left(\delta_{h}[f]\right)(x)=\left(\frac{1}{\cos \left(x+\frac{h}{2}\right)}-\cos \left(x+\frac{h}{2}\right)-\frac{1}{\cos \left(x-\frac{h}{2}\right)}\right)+\cos \left(x-\frac{h}{2}\right)$.

(81) Suppose for every $x$ holds $f(x)=$ ((the function tan) (the function $\cos ))(x)$ and $x_{0} \in \operatorname{dom}\left(\right.$ the function tan) and $x_{1} \in \operatorname{dom}$ (the function $\tan )$. Then $\Delta[f]\left(x_{0}, x_{1}\right)=\frac{\sin x_{0}-\sin x_{1}}{x_{0}-x_{1}}$.

(82) Suppose that

(i) for every $x$ holds $f(x)=(($ the function tan) (the function cos) $)(x)$,

(ii) $x \in \operatorname{dom}$ (the function tan), and 
(iii) $\quad x+h \in \operatorname{dom}$ (the function tan).

Then $\left(\Delta_{h}[f]\right)(x)=\sin (x+h)-\sin x$.

(83) Suppose that

(i) for every $x$ holds $f(x)=(($ the function tan) (the function cos) $)(x)$,

(ii) $x \in \operatorname{dom}$ (the function tan), and

(iii) $\quad x-h \in \operatorname{dom}$ (the function tan).

Then $\left(\nabla_{h}[f]\right)(x)=\sin x-\sin (x-h)$.

(84) Suppose that

(i) for every $x$ holds $f(x)=(($ the function tan) (the function cos) $)(x)$,

(ii) $\quad x+\frac{h}{2} \in \operatorname{dom}$ (the function $\tan$ ), and

(iii) $\quad x-\frac{h}{2} \in \operatorname{dom}$ (the function tan).

Then $\left(\delta_{h}[f]\right)(x)=\sin \left(x+\frac{h}{2}\right)-\sin \left(x-\frac{h}{2}\right)$.

(85) Suppose for every $x$ holds $f(x)=$ ((the function cot) (the function $\cos ))(x)$ and $x_{0} \in \operatorname{dom}$ (the function cot) and $x_{1} \in \operatorname{dom}$ (the function cot).

Then $\Delta[f]\left(x_{0}, x_{1}\right)=\frac{\left(\frac{1}{\sin x_{0}}-\sin x_{0}-\frac{1}{\sin x_{1}}\right)+\sin x_{1}}{x_{0}-x_{1}}$.

(86) Suppose that

(i) for every $x$ holds $f(x)=(($ the function cot) (the function $\cos ))(x)$,

(ii) $x \in \operatorname{dom}$ (the function cot), and

(iii) $x+h \in \operatorname{dom}$ (the function cot).

Then $\left(\Delta_{h}[f]\right)(x)=\left(\frac{1}{\sin (x+h)}-\sin (x+h)-\frac{1}{\sin x}\right)+\sin x$.

(87) Suppose that

(i) for every $x$ holds $f(x)=(($ the function cot) (the function $\cos ))(x)$,

(ii) $x \in \operatorname{dom}$ (the function cot), and

(iii) $\quad x-h \in \operatorname{dom}$ (the function cot).

Then $\left(\nabla_{h}[f]\right)(x)=\left(\frac{1}{\sin x}-\sin x-\frac{1}{\sin (x-h)}\right)+\sin (x-h)$.

(88) Suppose that

(i) for every $x$ holds $f(x)=(($ the function cot) (the function cos) $)(x)$,

(ii) $\quad x+\frac{h}{2} \in \operatorname{dom}$ (the function cot), and

(iii) $\quad x-\frac{h}{2} \in \operatorname{dom}$ (the function cot).

Then $\left(\delta_{h}[f]\right)(x)=\left(\frac{1}{\sin \left(x+\frac{h}{2}\right)}-\sin \left(x+\frac{h}{2}\right)-\frac{1}{\sin \left(x-\frac{h}{2}\right)}\right)+\sin \left(x-\frac{h}{2}\right)$.

(89) Suppose for every $x$ holds $f(x)=$ ((the function cot) (the function $\sin ))(x)$ and $x_{0} \in \operatorname{dom}$ (the function cot) and $x_{1} \in \operatorname{dom}$ (the function cot). Then $\Delta[f]\left(x_{0}, x_{1}\right)=\frac{\cos x_{0}-\cos x_{1}}{x_{0}-x_{1}}$.

(90) Suppose that

(i) for every $x$ holds $f(x)=(($ the function cot) (the function sin) $)(x)$,

(ii) $\quad x \in \operatorname{dom}$ (the function cot), and

(iii) $x+h \in \operatorname{dom}$ (the function cot).

Then $\left(\Delta_{h}[f]\right)(x)=\cos (x+h)-\cos x$.

(91) Suppose that

(i) for every $x$ holds $f(x)=(($ the function cot) (the function $\sin ))(x)$, 
(ii) $\quad x \in \operatorname{dom}$ (the function cot), and

(iii) $\quad x-h \in \operatorname{dom}$ (the function cot).

Then $\left(\nabla_{h}[f]\right)(x)=\cos x-\cos (x-h)$.

(92) Suppose that

(i) for every $x$ holds $f(x)=(($ the function cot) (the function $\sin ))(x)$,

(ii) $\quad x+\frac{h}{2} \in \operatorname{dom}$ (the function cot), and

(iii) $\quad x-\frac{h}{2} \in \operatorname{dom}$ (the function cot).

Then $\left(\delta_{h}[f]\right)(x)=\cos \left(x+\frac{h}{2}\right)-\cos \left(x-\frac{h}{2}\right)$.

(93) Suppose for every $x$ holds $f(x)=$ ((the function tan) (the function $\tan )(x)$ and $x_{0} \in \operatorname{dom}($ the function $\tan )$ and $x_{1} \in$ dom (the function $\tan )$. Then $\Delta[f]\left(x_{0}, x_{1}\right)=\frac{\left(\cos x_{1}\right)^{2}-\left(\cos x_{0}\right)^{2}}{\left(\cos x_{0} \cdot \cos x_{1}\right)^{2} \cdot\left(x_{0}-x_{1}\right)}$.

(94) Suppose that

(i) for every $x$ holds $f(x)=(($ the function tan) (the function tan) $)(x)$,

(ii) $x \in \operatorname{dom}$ (the function tan), and

(iii) $\quad x+h \in \operatorname{dom}$ (the function tan).

Then $\left(\Delta_{h}[f]\right)(x)=-\frac{\frac{1}{2} \cdot(\cos (2 \cdot(x+h))-\cos (2 \cdot x))}{(\cos (x+h) \cdot \cos x)^{2}}$.

(95) Suppose that

(i) for every $x$ holds $f(x)=(($ the function tan) (the function tan) $)(x)$,

(ii) $x \in \operatorname{dom}$ (the function tan), and

(iii) $\quad x-h \in \operatorname{dom}$ (the function tan).

Then $\left(\nabla_{h}[f]\right)(x)=-\frac{\frac{1}{2} \cdot(\cos (2 \cdot x)-\cos (2 \cdot(h-x)))}{(\cos x \cdot \cos (x-h))^{2}}$.

(96) Suppose that

(i) for every $x$ holds $f(x)=(($ the function tan) (the function tan $))(x)$,

(ii) $\quad x+\frac{h}{2} \in \operatorname{dom}$ (the function tan), and

(iii) $\quad x-\frac{h}{2} \in \operatorname{dom}$ (the function tan).

Then $\left(\delta_{h}[f]\right)(x)=-\frac{\frac{1}{2} \cdot(\cos (h+2 \cdot x)-\cos (h-2 \cdot x))}{\left(\cos \left(x+\frac{h}{2}\right) \cdot \cos \left(x-\frac{h}{2}\right)\right)^{2}}$.

\section{REFERENCES}

[1] Grzegorz Bancerek. The fundamental properties of natural numbers. Formalized Mathematics, 1(1):41-46, 1990.

[2] Czesław Byliński. Functions from a set to a set. Formalized Mathematics, 1(1):153-164, 1990.

[3] Czesław Byliński. Some basic properties of sets. Formalized Mathematics, 1(1):47-53, 1990.

[4] Krzysztof Hryniewiecki. Basic properties of real numbers. Formalized Mathematics, 1(1):35-40, 1990.

[5] Jarosław Kotowicz. Real sequences and basic operations on them. Formalized Mathematics, 1(2):269-272, 1990.

[6] Bo Li, Yan Zhang, and Xiquan Liang. Difference and difference quotient. Formalized Mathematics, 14(3):115-119, 2006, doi:10.2478/v10037-006-0014-z.

[7] Beata Perkowska. Functional sequence from a domain to a domain. Formalized Mathematics, 3(1):17-21, 1992.

[8] Konrad Raczkowski and Andrzej Nędzusiak. Series. Formalized Mathematics, 2(4):449452, 1991. 
[9] Andrzej Trybulec and Czesław Byliński. Some properties of real numbers. Formalized Mathematics, 1(3):445-449, 1990.

[10] Zinaida Trybulec. Properties of subsets. Formalized Mathematics, 1(1):67-71, 1990.

[11] Edmund Woronowicz. Relations defined on sets. Formalized Mathematics, 1(1):181-186, 1990.

[12] Yuguang Yang and Yasunari Shidama. Trigonometric functions and existence of circle ratio. Formalized Mathematics, 7(2):255-263, 1998.

Received November 17, 2009 